\title{
INTERSTELLAR DUST
}

\section{J. ALLAMANDOLA AND A. G. G. M. TIELENS (EDS.)}

Interstellar dust is of major importance in astrophysics because of its key roles in star and planet formation, interstellar chemistry, in determining the energy balance of gas clouds, and so on. The 40 invited reviews in this book bring the interested reader to the forefront of research in many different areas of dust research including observations, theory and laboratory experiments, and provide a doorway into the literature. There has been dramatic progress in all areas related to dust research during the last decade. In particular, the opening up of the IR window by ground-based and air- and space-borne observatories has provided a touchstone against which theories and experiments can be tested. A good example is the recent realization that polycyclic aromatic hydrocarbon molecules and related compounds may be a surprisingly ubiquitous component of the interstellar medium. Likewise, the isolation of largely unmodified interstellar grain components from meteorites and interplanetary dust particles promises to be equally revolutionary in the next decade as it broadens the purvue of this field to include direct laboratory studies of interstellar materials. The study of interstellar dust poses interesting challenges to astronomers, chemists and biochemists alike.

Coverphoto: () (1984) ROE/AAT Board. Photograph by David Malin from original plates taken with the UK Schmidt telescope, New South Wales, Australia.

\section{KLUWER ACADEMIC PUBLISHERS}

\author{
DORDRECHT / BOSTON / LONDON
}

